\title{
Effect of smoking history on outcome of patients diagnosed with TB and HIV
}

\author{
To the Editor: \\ Tobacco use, infection with HIV and active tuberculosis (TB) are important public health problems \\ worldwide. Smoking affects susceptibility to TB, with an increased risk of infection, TB disease and TB \\ death [1, 2]. An estimated 1.1 million of the 8.6 million people who developed TB in 2012 were \\ HIV-positive [3]. It has been estimated that smoking could cause 18 million excess cases of TB and \\ 40 million excess deaths between 2010 and 2050 [4], but little is known about the effect of smoking on the \\ outcomes of people receiving care for both HIV and TB.
}

We performed a secondary analysis of ITART (Integrated Tuberculosis and AntiRetroviral Treatment), a prospective cohort study of integrated TB/HIV care at primary care facilities in Kinshasa, Democratic Republic of Congo [5]. In this study, antiretroviral treatment (ART) was scheduled to start 1 month after TB treatment initiation if baseline CD4 cell count was $<100$ cells $\cdot \mathrm{mm}^{-3}$ or the patient had clinical stage 4 illness other than extrapulmonary $\mathrm{TB}$, and at 2 months if CD4 cell count was between 100 and 350 cells. $\mathrm{mm}^{-3}$ or if unavailable (e.g. if reagents were out of stock at the national HIV laboratory).

Associations between smoking and outcomes were assessed by multivariate Cox proportional hazard models and expressed as crude and adjusted hazard ratios (HR) and their 95\% confidence intervals. The outcomes of interest were uptake of ART during TB treatment and adverse TB treatment outcomes (lost to follow-up (LTFU), death or treatment failure). Follow-up started at TB treatment initiation and was censored at transfer out, adverse outcome or TB treatment completion (and ART initiation for the uptake analysis). Participants were classified as never-smokers or ever-smokers. Ever-smokers included current smokers, recent smokers (age of smoking cessation was the same as age at enrolment) and former smokers (age of smoking cessation was less than the age at enrolment). Among ever-smokers, duration was categorised as either greater or less than 10 years of smoking. The level of significance was set at $\mathrm{p}<0.05$. Analyses were carried out using SPSS 19.0.0 (IBM Corp., Armonk, NY, USA).

The study was approved by the ethics boards of the University of North Carolina at Chapel Hill (NC, USA) and the University of Kinshasa (DR Congo). All patients provided written informed consent.

A total of 599 patients diagnosed with TB and HIV were enrolled. Participants were excluded if they were $\leqslant 18$ years-old $(n=31)$ or if they were not ART-naïve $(n=36)$. Among the 533 participants included in the analysis, the median age was 38 years (IQR $31-45$ years) and $61 \%$ were female. Overall, $25 \%$ (135 out of 533) participants reported having ever smoked. Ever-smokers were older $(p=0.007)$ and a larger proportion of males (111 (51\%) out of 216) than females (24 (8\%) out of 317) reported any smoking $(\mathrm{p}<0.001)$. Among ever-smokers, 6\% (8 out of 135) reported current smoking, 25\% (34 out of 135) were recent smokers, and 69\% (93 out of 135) were former smokers. Among former smokers, 37\% (34 out of 93) stopped smoking $\geqslant 10$ years ago. Duration of smoking differed by sex, with $33 \%$ of males and $79 \%$ of females smoking for $<10$ years. Compared with those who never smoked, ever-smokers were less likely to have finished secondary education $(p=0.036)$, more likely to experience food insecurity $(p=0.006)$, and more likely to report risky behaviours including heavy drinking $(\mathrm{p}<0.001)$, sexual activity before 13 years of age $(\mathrm{p}=0.039)$ and multiple sexual partners ( $\mathrm{p}<0.001$ among females, $\mathrm{p}=0.745$ among males). Employment status, underweight, functional status and TB disease location did not differ significantly between ever-smokers and never-smokers.

Baseline CD4 cell count was available in 522 (98\%) participants. Median CD4 cell count was higher in current/recent smokers $\left(266\right.$ cells $\left.\cdot \mathrm{mm}^{-3}\right)$, compared with both never-smokers ( 166 cells $\left.\cdot \mathrm{mm}^{-3} ; \mathrm{p}=0.009\right)$ and former smokers $\left(162\right.$ cells $\left.\cdot \mathrm{mm}^{-3} ; \mathrm{p}=0.006\right)$. The same proportion $(79 \%)$ of never-smokers and ever-smokers had a baseline CD4 cell count $<350$ cells $\cdot \mathrm{mm}^{-3}$. Ever-smokers eligible for ART were $30 \%$ less likely to start ART during TB treatment (adjusted HR 0.70, 95\% CI 0.54-0.90). Among those initiating ART, adherence to ART was similar among ever- and never-smokers (68\% versus $77 \%$; $\mathrm{p}=0.131)$.

Adverse TB treatment outcomes were experienced by 19\% of participants: 11\% (56 out of 533) were LTFU, 9\% (46 out of 533) died during TB treatment and none experienced TB treatment failure. The proportion of individuals who experienced adverse TB treatment outcomes was higher in those who did not initiate ART (62 (37\%) out of 166) compared with those who started ART (40 (11\%) out of 367; p<0.001). Patients who were eligible for ART but failed to initiate ART were most likely to experience adverse TB treatment outcomes $(52(72 \%)$ out of 72$)$. Ever-smokers were about twice as likely to experience adverse 
TABLE 1 Tuberculosis (TB) treatment outcomes in relation to smoking history of HIV patients in Kinshasa, the Democratic Republic of Congo

\section{All patients $(n=533) \quad$ ART during TB No ART during TB} treatment $^{\#}(\mathrm{n}=367)$ treatment ${ }^{\#}(\mathrm{n}=166)$

\begin{tabular}{|c|c|c|c|}
\hline \multicolumn{4}{|l|}{ All ever-smokers ${ }^{\text {ๆ }}(n=135)$} \\
\hline Crude HR for LTFU & $2.43(1.43-4.13)$ & $2.05(0.86-4.88)$ & $2.17(1.11-4.26)$ \\
\hline Adjusted HR for LTFU & $2.40(1.13-5.11)$ & $2.77(0.95-8.08)$ & $1.67(0.70-3.97)$ \\
\hline Crude HR for death & $2.00(1.10-3.63)$ & $2.37(0.92-6.10)$ & $1.49(0.69-3.22)$ \\
\hline Adjusted HR for death & $0.87(0.38-1.98)$ & $1.50(0.48-4.67)$ & $1.08(0.19-6.33)$ \\
\hline Crude HR for adverse outcome ${ }^{+}$ & $2.15(1.45-3.20)$ & $2.14(1.13-4.06)$ & $1.73(1.05-2.87)$ \\
\hline Adjusted HR for adverse outcome ${ }^{+}$ & $1.57(1.00-2.47)$ & $2.21(1.16-4.22)$ & $1.15(0.46-2.88)$ \\
\hline \multicolumn{4}{|l|}{ Duration of smoking } \\
\hline \multicolumn{4}{|l|}{ Smoked >10 years ${ }^{11}(n=79)$} \\
\hline Crude HR for LTFU & $2.89(1.58-5.26)$ & $2.46(0.89-6.84)$ & $2.29(1.08-4.84)$ \\
\hline Adjusted HR for LTFU & $2.71(1.01-7.28)$ & $5.11(0.96-27.06)$ & $1.37(0.52-3.60)$ \\
\hline Crude HR for death & $2.93(1.55-5.54)$ & $4.55(1.76-11.73)$ & $1.62(0.68-3.88)$ \\
\hline Adjusted HR for death & $1.64(0.63-4.26)$ & $3.81(0.99-14.58)$ & $0.80(0.11-5.53)$ \\
\hline Crude HR for adverse outcome ${ }^{+}$ & $2.79(1.80-4.31)$ & $3.28(1.65-6.52)$ & $1.84(1.05-3.25)$ \\
\hline Adjusted HR for adverse outcome ${ }^{+}$ & $2.39(1.36-4.22)$ & $4.62(2.09-10.21)$ & $1.04(0.39-2.73)$ \\
\hline \multicolumn{4}{|l|}{ Smoked $<10$ years $s^{\pi}(n=56)$} \\
\hline Crude HR for LTFU & $1.84(0.85-4.00)$ & $1.58(0.46-5.51)$ & $1.98(0.74-5.34)$ \\
\hline Adjusted HR for LTFU & $3.04(1.23-7.53)$ & $1.74(0.44-6.95)$ & \\
\hline Crude HR for death & $0.72(0.25-2.65)$ & $0.04(0.00-143.00)$ & $1.24(0.37-4.22)$ \\
\hline Adjusted HR for death & $0.41(0.09-1.75)$ & $\S$ & $0.38(0.04-3.69)$ \\
\hline Crude HR for adverse outcome ${ }^{+}$ & $1.33(0.70-2.52)$ & $0.89(0.27-2.96)$ & $1.52(0.71-3.28)$ \\
\hline Adjusted HR for adverse outcome ${ }^{+}$ & $1.28(0.67-2.44)$ & $0.87(0.25-3.00)$ & $1.45(0.66-3.15)$ \\
\hline \multicolumn{4}{|l|}{ Duration since exposure } \\
\hline \multicolumn{4}{|l|}{ Current and recent smokers" ( $\mathrm{n}=42)$} \\
\hline Crude HR for LTFU & $2.70(1.24-5.85)$ & $2.07(0.47-9.12)$ & $1.96(0.78-4.94)$ \\
\hline Adjusted HR for LTFU & 3.09 (1.01-9.39) & $3.70(0.47-29.21)$ & $1.51(0.30-7.62)$ \\
\hline Crude HR for death & $3.15(1.44-6.89)$ & $4.11(1.15-14.73)$ & $1.81(0.67-4.87)$ \\
\hline Adjusted HR for death & $2.03(0.70-5.93)$ & $3.93(0.63-24.33)$ & $1.27(0.18-9.28)$ \\
\hline Crude HR for adverse outcome ${ }^{+}$ & $2.77(1.60-4.81)$ & $2.87(1.10-7.50)$ & $1.77(0.90-3.49)$ \\
\hline Adjusted HR for adverse outcome ${ }^{+}$ & $2.71(1.42-5.16)$ & $3.75(1.00-14.10)$ & $1.48(0.49-4.52)$ \\
\hline \multicolumn{4}{|l|}{ Former smokers ${ }^{\pi}(n=93)$} \\
\hline Crude HR for LTFU & $2.32(1.27-4.22)$ & $2.03(0.78-5.29)$ & $2.34(1.08-5.06)$ \\
\hline Adjusted HR for LTFU & $2.62(1.19-5.75)$ & $2.09(0.70-6.18)$ & $1.77(0.47-6.66)$ \\
\hline Crude HR for death & $1.50(0.71-3.18)$ & $1.79(0.57-5.62)$ & $1.26(0.47-3.38)$ \\
\hline Adjusted HR for death & $0.53(0.19-1.48)$ & $1.22(0.33-4.48)$ & $0.16(0.02-1.31)$ \\
\hline Crude HR for adverse outcome ${ }^{+}$ & $1.88(1.18-2.99)$ & $1.89(0.91-3.94)$ & $1.70(0.93-3.10)$ \\
\hline Adjusted HR for adverse outcome ${ }^{+}$ & $1.25(0.74-2.12)$ & $1.92(0.92-3.99)$ & $0.92(0.34-2.50)$ \\
\hline \multicolumn{4}{|c|}{$\begin{array}{l}\text { Data are presented as estimate }(95 \% \mathrm{Cl}) \text {, unless otherwise stated. Analysis was performed by Cox } \\
\text { proportional hazard modelling using a } 10 \% \text { change in estimate approach for the covariates sex, age } \\
\text { (quintiles), underweight, functional status lambulatory versus in bed more than usual), CD4 cell count } \\
\text { category }\left(<50,50-99,100-350,>350 \text { cells } \mathrm{mm}^{-3}\right) \text {, ART initiation during TB treatment, extrapulmonary TB, } \\
\text { completion of secondary education, employment, food insecurity (hungry in the past week), multiple sexual } \\
\text { partners (excluded for the mortality analysis), sexual activity before age } 13 \text { years of age (excluded for the } \\
\text { mortality analysis), and heavy alcohol consumption (World Health Organisation } 2014 \text { definition [6]). ART: } \\
\text { antiretroviral treatment; HR: hazard ratio; LTFU: lost to follow-up. \#: ART initiated within } 6 \text { months } \\
\text { following start of TB treatment; }{ }^{\uparrow} \text { : compared to patients who never smoked; }{ }^{+}: \text {mortality and loss to } \\
\text { follow-up during TB treatment combined; }{ }^{\S} \text { : number of patients too low to perform this analysis. }\end{array}$} \\
\hline
\end{tabular}

TB treatment outcomes compared with never-smokers (crude HR 2.15, 95\% CI 1.45-3.20) (table 1). The association was significant after adjustment for covariates among all patients (adjusted HR 1.57, 95\% CI 1.00-2.47) and people on ART (adjusted HR 2.21, 95\% CI 1.16-4.22), but not among those who did not initiate ART during TB treatment (adjusted HR 1.15, 95\% CI 0.46-2.88). When examining the type of adverse TB treatment outcome, LTFU was independently associated with smoking status (adjusted HR 2.40, 95\% CI 1.13-5.11), but death was not (adjusted HR 0.87, 95\% CI 0.38-1.98).

When stratified by duration of smoking, smoking was independently associated with adverse TB treatment outcome among those reporting smoking for $\geqslant 10$ years (adjusted HR 2.39, 95\% CI 1.36-4.22) but not 
among those smoking for $<10$ years (adjusted HR 1.28, 95\% CI 0.67-2.44). When further stratifying by ART status during TB treatment, patients who had smoked for $\geqslant 10$ years and initiated ART during TB treatment were five times as likely to be LTFU (adjusted HR 5.11,95\% CI 0.96-27.06) and almost four times as likely to die (adjusted HR 3.81, 95\% CI 0.99-14.58) compared with never-smokers who initiated ART.

Finally, those who reported recent or current smoking were nearly three times as likely to experience adverse TB treatment outcomes compared with those who had never smoked (adjusted HR 2.71, 95\% CI $1.42-5.16)$.

Few studies have assessed smoking as a risk factor for adverse care outcomes in people living with TB and HIV. Regarding LTFU, individuals in Brazil [7] who smoked in the 6 months preceding TB treatment were twice as likely to be LTFU (adjusted OR 2.62, 95\% CI 1.31-5.26). In Thailand [8], current smoking was also associated with twice the odds of LTFU (adjusted OR 2.3, 95\% CI 1.3-4.1). Regarding mortality, a study from Iran [9] reported no effect of smoking, but only included three participants who had never smoked. In Brazil [10], current smokers hospitalised for TB, $71 \%$ of whom were HIV infected and were predominantly ART-naïve, were at increased risk of death (adjusted OR 2.14, 95\% CI 1.07-4.28), but estimates were not stratified by HIV or ART status.

In summary, we observed that ever-smokers eligible for ART were 30\% less likely to initiate ART during TB treatment, and ever-smokers on ART were twice as likely to experience adverse TB treatment outcomes. The effect of smoking was most pronounced among those on ART who were current/recent smokers or those who had smoked for $\geqslant 10$ years. ART was a strong effect modifier, with the absence of an effect of smoking on adverse TB treatment outcomes likely due to the strong effect of the absence of ART on death and LTFU in TB patients. While tobacco smoke has well-known hazardous effects, smokers also have a different psychosocial risk profile, which could contribute to the observed relationship between smoking and adverse TB treatment outcomes. Our estimates were, however, adjusted for many of these psychosocial factors.

In addition to preventing people from smoking, helping smokers to quit and providing ART, the outcome of people living with HIV who develop TB might be improved by increasing support for (former) smokers, such as intensified counselling on the importance of ART.

- @ERSpublications

Among TB-HIV patients on antiretrovirals those who smoke(d) are more likely to have adverse TB treatment outcomes http://ow.ly/DyPqW

Koen Vanden Driessche ${ }^{1-3}$, Monita R. Patel ${ }^{1}$, Nana Mbonze ${ }^{4}$, Martine Tabala ${ }^{4}$ Marcel Yotebieng ${ }^{1,4,5}$, Frieda Behets ${ }^{1}$ and Annelies Van Rie ${ }^{1}$

${ }^{1}$ Dept of Epidemiology, University of North Carolina at Chapel Hill, Chapel Hill, NC, USA. ${ }^{2}$ Division of Infectious and Immunological Diseases, Dept of Paediatrics, British Columbia Children's Hospital, Centre for Understanding and Preventing Infection in Children, Child and Family Research Institute, University of British Columbia, Vancouver, BC, Canada. ${ }^{3}$ The Nijmegen Institute for Infection, Inflammation, and Immunity, Radboud University Medical Centre, Nijmegen, The Netherlands. ${ }^{4}$ School of Public Health, University of Kinshasa, Kinshasa, DR Congo. ${ }^{5}$ College of Public Health, Ohio State University, Columbus, OH, USA.

Correspondence: Correspondence: Annelies Van Rie, 2104F McGavran Greenberg Hall, 27599-7534 Chapel Hill, NC, USA. E-mail: Vanrie@email.unc.edu

For editorial comments see Eur Respir J 2015; 45: 583-585 [DOI: 10.1183/09031936.00221814].

Received: June 242014 | Accepted after revision: Oct 222014 | First published online: Nov 272014

Support statement: This study was funded by the US Centers for Disease Control and Prevention (UNC-GAP project: CDC U62 CCU422), US President's Emergency Plan for AIDS Relief (PEPFAR), and The Global Fund to Fight AIDS, Tuberculosis and Malaria. K. Vanden Driessche is supported by an ESPID Fellowship Award and M.R. Patel by an NIH training grant 2T32AI070114.

Conflict of interest: Disclosures can be found alongside the online version of this article at erj.ersjournals.com

Acknowledgements: The authors thank the participating healthcare workers. Without their enthusiasm and dedication, this work could not have been carried out. We thank S. Mpuate (School of Public Health, University of Kinshasa, Kinshasa, DR Congo) and E. Cromwell (Dept of Epidemiology, University of North Carolina, Chapel Hill, NC, USA) for assistance in preparing the ITART dataset and J. Bettinger (Vaccine Evaluation Center, University of British Columbia, Vancouver, BC, Canada) for helpful discussions.

\section{References}

1 Lin HH, Ezzati M, Murray M. Tobacco smoke, indoor air pollution and tuberculosis: a systematic review and meta-analysis. PLoS Med 2007; 4: e20.

2 van Zyl Smit RN, Pai M, Yew WW Global lung health: the colliding epidemics of tuberculosis, tobacco smoking, HIV and COPD. Eur Respir J 2010; 35: 27-33. 
3 World Health Organization. Global tuberculosis report 2013. Geneva, WHO Press, 2013.

4 Basu S, Stuckler D, Bitton A Projected effects of tobacco smoking on worldwide tuberculosis control: mathematical modelling analysis. BMJ 2011; 343: d5506.

5 Van Rie A, Patel MR, Nana M Integration and task-shifting for TB/HIV care and treatment in highly resource-scarce settings: one size may not fit all. J Acquir Immune Defic Syndr 2014; 65: e110-e117.

World Health Organization. Global status report on alcohol and health. Geneva, WHO Press, 2014.

Maruza M, Albuquerque MF, Coimbra I Risk factors for default from tuberculosis treatment in HIV-infected individuals in the state of Pernambuco, Brazil: a prospective cohort study. BMC Infect Dis 2011; 11: 351.

8 Kittikraisak W, Burapat C, Kaewsa-ard S Factors associated with tuberculosis treatment default among HIV-infected tuberculosis patients in Thailand. Trans R Soc Trop Med Hyg 2009; 103: 59-66.

9 Tabarsi P, Chitsaz E, Moradi A Treatment outcome, mortality and their predictors among HIV-associated tuberculosis patients. Int J STD AIDS 2012; 23: e1-e4.

10 Silva DR, Menegotto DM, Schulz LF Factors associated with mortality in hospitalized patients with newly diagnosed tuberculosis. Lung 2010; 188: 33-41.

\title{
Pulmonary hypertension associated with obliterative phlebitis in IgG4-related lung disease
}

\author{
To the Editor:
}

IgG4-related disease (IgG4-RD) is a systemic fibroinflammatory disease associated with elevated serum IgG4 level and IgG4-positive plasma cell infiltration into multiple organs [1]. In 2001, Hamano et al. [2] reported that patients with autoimmune pancreatitis have high-serum IgG4 concentrations. Subsequently, IgG4-related lesions similar to those of autoimmune pancreatitis have been identified in other organs [1] including the lungs [3].

Thoracic involvement in IgG4-RD has been increasingly recognised, usually manifesting as inflammatory pseudotumours, interstitial pneumonia or occasionally, pleuritis [3]. However, little information is available on airway disease or pulmonary hypertension $(\mathrm{PH})$ associated with IgG4-RD. In this report, we describe a patient presenting with $\mathrm{PH}$ associated with IgG4-related bronchiolitis.

A 45-year-old male first presented with community-acquired pneumonia caused by Haemophilus influenzae. When he was discharged, slight dyspnoea on exertion and abnormal findings in computed tomography (CT) of the chest remained. After 5 months of follow-up, he was admitted again for a close inspection of deteriorating dyspnoea, which was categorised as World Health Organization class II. He had a 10 -year history of refractory sinusitis. For the last 5 years, he had complained of swelling of the bilateral eyelids, which was diagnosed as "nonspecific dacryoadenitis" based on left lacrimal grand biopsy at a previous hospital clinic visit. He was an ex-smoker (one pack per day for 20 years) and had no relevant occupational or environmental exposures.

A chest CT scan manifested diffuse bilateral centrilobular nodules, prominent peripheral pulmonary vascular markings (fig. 1a) and mediastinal lymphadenopathy with no evidence of pulmonary large vessel compression (fig. 1b). Laboratory examinations showed high serum IgG $\left(6530 \mathrm{mg} \cdot \mathrm{dL}^{-1}\right)$ and $\operatorname{IgG} 4$ $\left(>1500 \mathrm{mg} \cdot \mathrm{dL}^{-1}\right.$ ) levels. Serum C-reactive protein, lactate dehydrogenase, Krebs von den Lungen-6 antigen and surfactant protein-D levels were normal. Results of evaluations for connective tissue diseases, angiotensin converting enzyme, human leukocyte antigen-B54 and human T-lymphotropic virus-1 antigen were all negative. Arterial blood gas showed normal partial pressure of oxygen and carbon dioxide (87.5 and 35.3 Torr, respectively). A pulmonary function test indicated obstructive disorder with decreased forced expiratory volume in $1 \mathrm{~s}$ (FEV1)/forced vital capacity ratio (FVC) and decreased predicted value for diffusing capacity of the lung for carbon monoxide (\%DLCO) (FEV1/FVC 67.3\%; \%DLCO 73.6\%). The predicted values for FVC (\%FVC) and FEV1 (\%FEV1) were normal (\%FVC 108.4\%; \%FEV1 81.6\%). Bronchoalveolar lavage fluid cell count was $0.32 \times 10^{5}$ cells $\cdot \mathrm{mL}^{-1}$, with a normal cellular profile and CD4/ CD8 ratio. Microbial culture results on the sputum and bronchoalveolar lavage fluid were negative. Although echocardiography disclosed no abnormalities, pulmonary arterial catheterisation revealed elevated pulmonary arterial pressure (PAP) (systolic $47 \mathrm{mmHg}$, diastolic $23 \mathrm{mmHg}$, mean $33 \mathrm{mmHg}$ ) 